\title{
A new class of spontaneously polarized materials
}

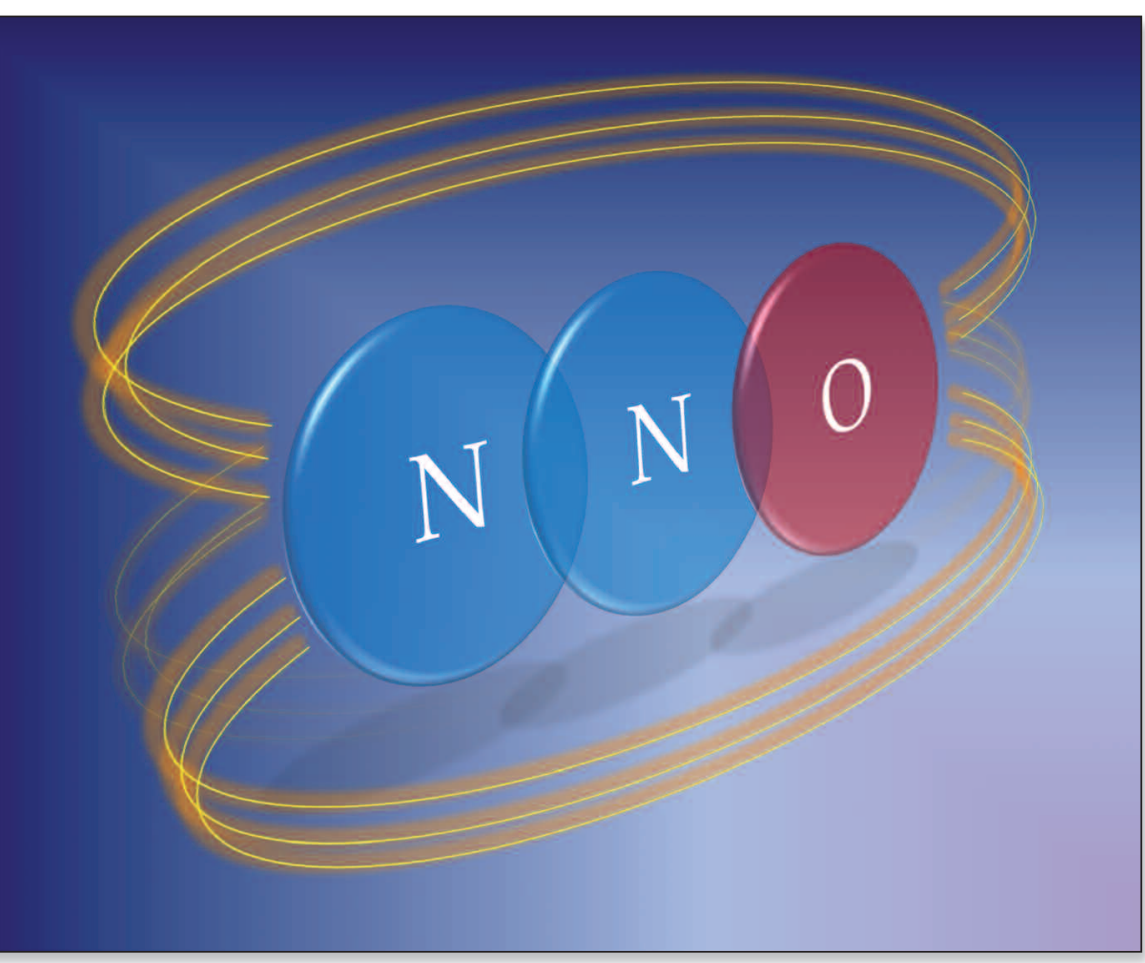

aterials have been a defining feature of the evolution of human culture and the electrical properties of materials are now at the forefront of this evolution. Advances occur both through the discovery of new electrical characteristics and through new devices based upon these discoveries. Here we describe a hitherto unsuspected electrical property of materials.

We have recently found that solid thin films of many common or garden molecules harbour very large electric fields [1]. These fields are a property of the film and appear spontaneously when films of species such as nitrous oxide or isoprene are laid down on a substrate. The fields point in a direction perpendicular to the surface and may exceed $10^{8} \mathrm{~V} / \mathrm{m}$. The films can be as thin as ten monolayers for large electric fields to be established. This new class of ferroelectric-like materials are of the order-disorder type, as opposed to displacive ferroelectrics such as the class of perovskites [2], in which fields are generated through small displacements of lattice atoms from their positions in the non-ferroelectric phase. We are hesitant to name the present films simply 'ferroelectric' since, while they satisfy the requirement that they harbour spontaneous macroscopic electric
- David Field, Oksana Plekan, Andrew Cassidy, Richard Balog and Nykola Jones

Department of Physics and Astronomy (IFA) and Institute for Storage Ring Facilities (ISA)

University of Aarhus, Aarhus 8000 C, Denmark

DOI: 10.1051/epn/2011605

Very large electric fields form spontaneously within films of seemingly prosaic chemicals such as nitrous oxide or propane. We describe how the discovery of this unexpected phenomenon took place and how we attempt to understand the nature of the new class of spontaneously polarized materials, resembling ferroelectrics, which these observations herald.

fields, we have not shown that these fields can be reversed by application of an external field. Note also that the films which we describe are not 'electrets', which possess a fixed charge or ordered dipole orientation induced by charge injection or an externally imposed electrostatic field.

\section{The experimental method}

The peculiar nature of films described below makes itself felt by the spontaneous appearance of a voltage on the surface. The experimental challenge is to measure this voltage without perturbing it significantly. The electric field within the film is then given by the measured voltage divided by the film thickness.

In our experiment this is achieved as follows. A beam of low energy electrons, formed by synchrotron radiation photoionization of Ar, travelling through vacuum at energies down to two to three $\mathrm{meV}$ with an energy resolution of $1.5 \mathrm{meV}$, is directed onto a film surface (figure 1). Electrons are formed at a nominal zero potential with an energy of a few meV and should therefore just be able to reach a target when the target is itself at a nominal zero. If the target were not at zero but rather, say, at +5 volts due to a spontaneous formation of an electric field within 
the film, then in order to ensure that the electrons only just reach the target it would be necessary to bias the target 5 volts negative. Measurement of the bias required to locate the onset of a measureable current, that is, 1 to 2 femtoamps, therefore gives the potential on the surface of the film, some small corrections apart. The necessary bias is measured by connecting a highly sensitive ammeter (a 'femtoammeter': figure 1) to the gold substrate on which films are laid down.

\section{Еuрпка}

Our discovery of the electrical properties of our films was serendipitous. We had chosen to observe electron scattering at very low energy in a $40 \mathrm{~K}$ film of $\mathrm{N}_{2} \mathrm{O}$, a molecule selected since it is one of the basic molecules of chemistry in the natural world. We found that a current was detected at the femtoammeter which remained a large proportion of the incident current when the film voltage was set equal to zero, the nominal potential at which the electrons were formed (see Figure 1). A negative bias was applied to the sample to attempt to reduce this current to zero. We discovered that we needed to apply more than 2 volts to achieve this. The apparatus was designed to measure millivolt shifts in order to record negative charging of the target due to electron capture in the film, as for example in water ice [3]. Here we required a bias of $>2 \mathrm{~V}$ and in the wrong sense, that is, negative rather than positive. Hence our surprise.

Our first thought was that our experimental apparatus was malfunctioning and was recording a leakage current through imperfect insulation to ground. A few simple tests showed that this was not the case; for example we found that increasing the thickness of the layer of $\mathrm{N}_{2} \mathrm{O}$ increased the voltage on the surface of the film almost proportionally. In due course we found that 1253 monolayers (ML), equivalent to $0.4 \mu \mathrm{m}$, of $\mathrm{N}_{2} \mathrm{O}$ laid down at $40 \mathrm{~K}$ could support a voltage of $38.40 \mathrm{~V}$. Here we stopped for fear of wrecking our femtoammeter. The measured voltages also showed a strong dependence on the temperature at which films were laid down, creating much higher voltages for lower temperatures for any given film thickness. This is shown in figure 2 for ten different temperatures between $38 \mathrm{~K}$ and $65 \mathrm{~K}$. Electric fields were correspondingly stronger at lower temperatures. For example, a film laid down at $38 \mathrm{~K}$ showed a field of $1.19 \pm 0.08 \times 10^{8} \mathrm{~V} / \mathrm{m}$ in the $\mathrm{N}_{2} \mathrm{O}$ film, dropping to $1.60 \pm 0.1 \times 10^{7} \mathrm{~V} / \mathrm{m}$ at $65 \mathrm{~K}$ (figure 3 ). Fields were calculated using 1 monolayer $(\mathrm{ML})=0.32 \mathrm{~nm}$.

Why do films not break down electrically in the presence of such large electric fields? For comparison, the breakdown of dry air occurs at electric fields of a few times $10^{6} \mathrm{~V} / \mathrm{m}$, while the field in a spark plug to ignite petrol in an internal combustion engine is around $2 \times 10^{7} \mathrm{~V} / \mathrm{m}$. However, in solids in general, breakdown fieldsiv vary between $10^{8} \mathrm{~V} / \mathrm{m}$ to $10^{9} \mathrm{~V} / \mathrm{m}$, where

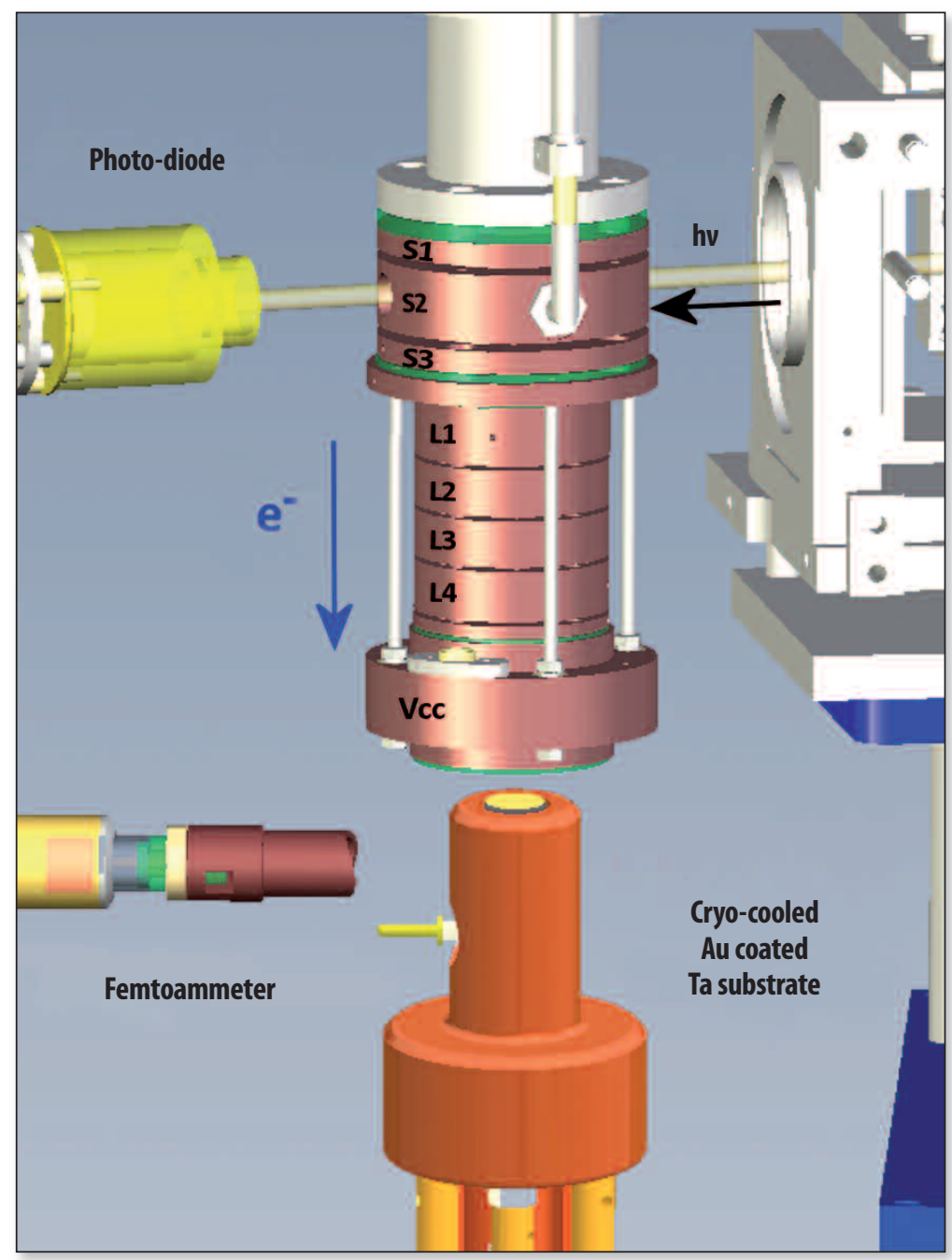

$\Delta$ FIG. 1: Detection of surface potentials using electron beams: electrons are formed in the gas phase through threshold photoionization of Argon at $\sim 15.75 \mathrm{eV}$, using monochromatic synchrotron radiation ' $h v$ ' at a resolution of $\sim 1.5 \mathrm{meV}$. Electrons are created at the potential of S2, which is set to a nominal zero, and directed under Ultra-high vacuum (UHV) conditions onto condensed solid films at temperatures of $38 \mathrm{~K}$ and above. Current is detected with a femtoammeter. We use the ASTRID synchrotron radiation source at the University of Aarhus, where all the experiments described here were conducted. Radiation not absorbed by Argon is collected at the photodiode.

electromechanical stress is induced by elastic deformation and electron cascade. The latter will be inhibited by inelastic scattering of electrons within solid films. Given that it is possible for such large fields to be present in stable solids, other issues come to mind. What is the nature of the interactions which give rise to such powerful electric fields within the medium? Have we stumbled upon a new class of materials of which $\mathrm{N}_{2} \mathrm{O}$ is but one member? Is the nature of the substrate, polycrystalline gold in this case, of significance for the phenomenon to occur?

\section{POSITIVE AND NEGATIVE}

Films of different molecules can carry a positive charge on their surface ( $\mathrm{N}_{2} \mathrm{O}$, isoprene, toluene) or a negative (propane, isopentane, $\mathrm{CF}_{3} \mathrm{Cl}$ ). The value of the electric field within the material may also differ greatly from case to case. Both features are illustrated by data in Figure 4. 


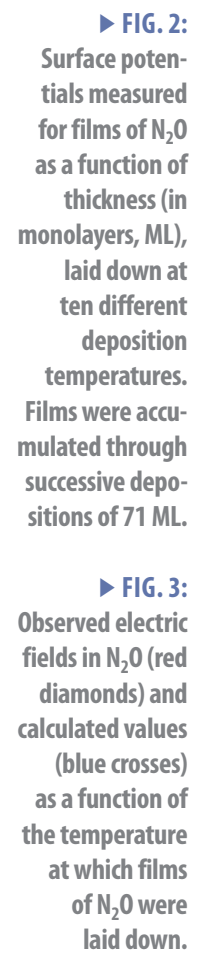

\section{Film deposition temperature: a model}

Our hypothesis is that the mechanism for the creation of electric fields in the film is due to a tendency of the molecular dipoles to line up, plus to minus, in successive layers of the material. At the surface-vacuum interface, the positive nitrogen end of $\mathrm{N}_{2} \mathrm{O}$, which has the structure $\left(\delta^{+}\right) \mathrm{N}-\mathrm{N}-\mathrm{O}\left(\delta^{-}\right)$, protrudes out of the surface and the resulting charge density on the surface gives rise to polarization and a positive voltage. Thus, polarization resulting from dipole alignment gives rise to the electric field, rather than free charges. This is a non-linear system since alignment of dipoles creates the field and the field itself creates dipole alignment. Since there is negligible charge within the film, Gauss' theorem requires that the field be constant.

The reason why a strong temperature dependence of the electric field on deposition temperature is observed (figure 3 ) is that dipole ordering is countered by a tendency to disorder through thermal motion. The control parameter of the physics is therefore the energy associated with the interaction of the dipole with the local electric field divided by the thermal energy.

The non-linear order-disorder problem may be solved by methods set out by Langevin, Debye and Landau, using so-called mean field theory. Solutions yield the average direction in which a dipole is pointing within the film, relative to the normal to the film surface, as a function of temperature. This in turn gives the amount of charge at the surface-vacuum interface per unit area - the polarization - and hence the electric field. Theoretical values are fitted to observed data of electric field versus temperature, using three temperature-independent

\section{CURIE POINTS}

A critical temperature at which films lose their electric fields, can be observed for isoprene, isopentane and propane, for which spontaneous polarisation is lost by heating whilst the film survives. This makes the analogy with ferroelectric materials where a similar phenomenon is observed. parameters and yielding results shown as blue crosses in figure 3. The good agreement between this theory albeit parameterized - and observations gives strong support to the hypothesis that dipole alignment is the cause of the unexpected electrical nature of the films. Note also that dipoles are not pointing vertically but rather are typically aligned at an angle of more than $80^{\circ}$ to the normal.

\section{A new class of spontaneously polarized material}

We have recently tested seven species for the presence of a spontaneous electric field, the only criterion of choice being that they should possess low dipole moments. Of these the first six, propane (dipole moment $0.08 \mathrm{D})$, isopentane (0.13D), $\mathrm{N}_{2} \mathrm{O}(0.167 \mathrm{D})$, isoprene (0.25D), toluene (0.385D) and $\mathrm{CF}_{3} \mathrm{Cl}$ (Freon13; 0.5D) all form polarized films. The seventh, OCS, with a dipole moment of $0.7 \mathrm{D}$, does not - nor does $\mathrm{H}_{2} \mathrm{O}$ with a still larger dipole moment of $1.85 \mathrm{D}$.

It appears a reasonably safe conclusion that all species with dipole moments between close to zero and $0.5 \mathrm{D}$, and lacking hydrogen bonding, form polarized layers. We therefore suggest that we have discovered a new class of polarized materials. To emphasise the generality of the new phenomenon, $\mathrm{N}_{2} \mathrm{O}$ has been laid down on 20ML of xenon. Exactly the same values of the electric field are found as for films laid down on amorphous gold.

Different molecules have, of course, different properties. The most striking difference is that some species carry a positive charge on their surface $\left(\mathrm{N}_{2} \mathrm{O}\right.$, isoprene, toluene) and some a negative (propane, isopentane, $\mathrm{CF}_{3} \mathrm{Cl}$ ). The value of the electric field within the material may also differ greatly from case to case. This is shown in figure 4 , expressing the electric field in terms of $\mathrm{mV}$ of potential increase upon the addition of 1 monolayer (ML).

The values shown in figure 4 are highly reproducible. However, we have found some complexity in the case of propane. Films are built up by successive deposition and for propane we found typically that thinner layers 
showed $\sim 0.7 \mathrm{mV} / \mathrm{ML}$ whereas thicker layers of $>3700 \mathrm{ML}$ appeared to switch phase and yield $4.77 \mathrm{mV} / \mathrm{ML}$. Both these values are shown in figure 4.

There is an optimum dipole moment for creation of polarized films, around $0.25 \mathrm{D}$. No quantitative explanation can be offered for this value at present. At values of the dipole $>0.5 \mathrm{D}$ the tendency of molecules to align with positive and negative ends close to each other overcomes the tendency to create order leading to net polarisation, at any rate in the absence of other influences such as hydrogen bonding. The result is then zero net polarization and zero electric field within the film.

Each of the species in figure 4 shows similar strong temperature dependence of the electric field as that found in $\mathrm{N}_{2} \mathrm{O}$. For example, a $280 \mathrm{ML}$ film of isoprene laid down at $40 \mathrm{~K}$ shows a surface potential of $9 \mathrm{~V}$ whereas if laid down at $70 \mathrm{~K}$ the value is $1.80 \mathrm{~V}$. Potentials may also be observed at high temperatures: for example toluene shows $\sim 2 \mathrm{~V}$ at $115 \mathrm{~K}$.

Polarized films are very robust to temperature stress. For example a $426 \mathrm{ML}$ film of $\mathrm{N}_{2} \mathrm{O}$ was laid down at $38 \mathrm{~K}$ and heated in steps of $3.7 \mathrm{~K}$ over a period of 90 minutes to just below the evaporation point at $75 \mathrm{~K}$. The surface potential was measured at each temperature. The potential drops by only $510 \mathrm{meV}$. This may be compared with data in Figure 1 where results for the fully relaxed state show a drop of $11.95 \mathrm{~V}$ between $38 \mathrm{~K}$ and $65 \mathrm{~K}$. It has also been found that deposition above the Curie temperature and reduction of the temperature to below the Curie temperature does not lead to the establishment of an electric field within the layer.

\section{Concluding comments}

The discovery of this new class of spontaneously polarized materials opens up a wide field of possible applications. These may arise rather generally in nanoxerography and voltage-patterned surfaces could for example be employed to create very high-density biosensor arrays. Moreover, it turns out to be possible to manipulate these films very readily to create a broad range of electric field structures. Presently we seek materials which can be used under less extreme cryoconditions and this will be one focus of future research. Other future areas may be effects of rate of deposition of films, dilution of the active material in xenon and the creation of more complex electrical nanostructures. We have also very recently found that some higher dipole moment species such as methyl formate (1.75 D) and 2,5, dihydrofuran (1.53 D) can spontaneously form polarized films. This is under further investigation.

\section{About the Authors}

David Field has been a professor of Physics and Astronomy at Aarhus for the last decade or so and is scientific director of the Institute for Storage Ring Facilities (ISA).

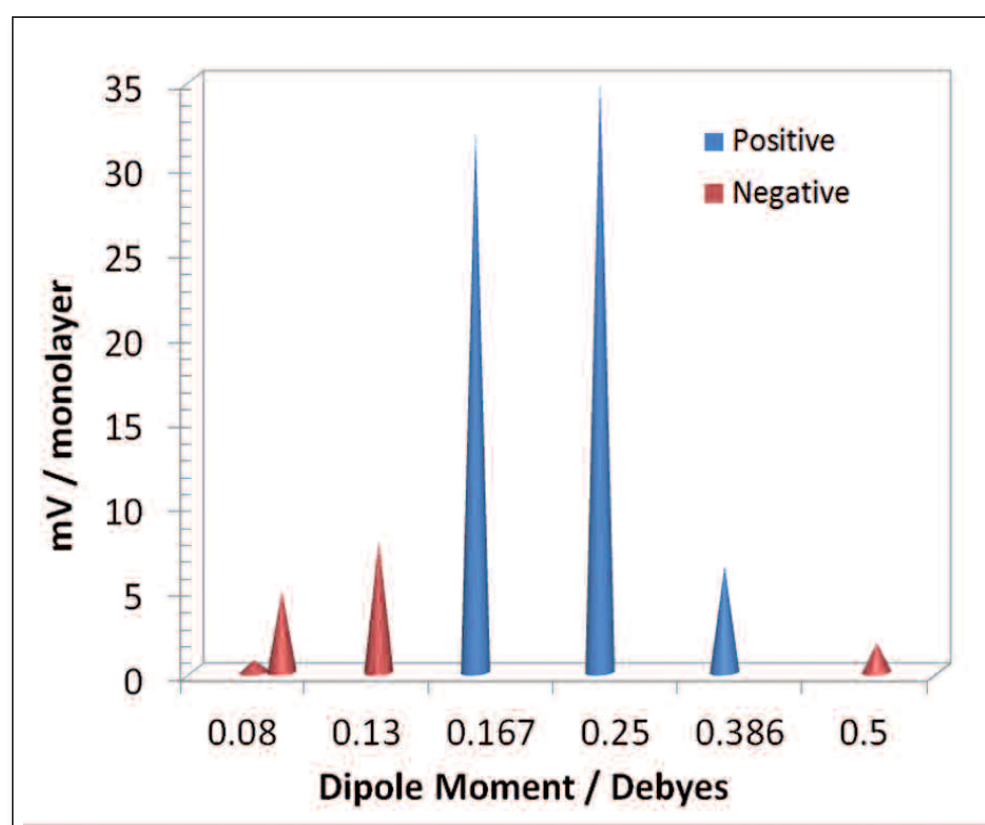

Oksana Plekan is an experienced post-doc, funded by $\triangle$ FIG. 4: the Danish Research Council, who worked for several The electric field in terms of $\mathrm{mV}$ of potential increase upon the addition of 1 monolayer (ML) of different compounds. Values given are for layers laid down at $40 \mathrm{~K}$. Here $0.08 \mathrm{D}$ refers to propane, 0.13D to isopentane, $0.167 \mathrm{D}$ to nitrous oxide, $0.25 \mathrm{D}$ to isoprene, $0.385 \mathrm{D}$ to toluene and $0.50 \mathrm{D}$ to $\mathrm{CF}_{3} \mathrm{Cl}$. Two figures are given for propane (see text). Red cones represent a negative potential on the surface, blue a positive potential.

\section{QUANTUM WELLS}

Quantum wells can be can be readily assembled. A 33ML layer of $\mathrm{N}_{2} \mathrm{O}$ was laid down at $40 \mathrm{~K}$ generating $+800 \mathrm{mV}$ at the film surface. Subsequently, $183 \mathrm{ML}$ of isopentane was laid directly on top of the $\mathrm{N}_{2} \mathrm{O}$ and created precisely $-800 \mathrm{mV}$, bringing the potential on the film surface back to zero, forming a $2 \mathrm{D}$ triangular quantum well. The possibilities are manifold: for example, Xe spacers can be used to form flat-bottomed wells. With standard masking techniques a wide range of nanostructures could be formed, for example quantum dots. 